\title{
Effect of the parasympathetic vasodilation on temperature regulation via trigeminal afferents in the orofacial area
}

\author{
Hanako Ohke ${ }^{1}$, Toshiya Sato ${ }^{2}$, Kohei Mito ${ }^{2}$, Makoto Terumitsu ${ }^{1}$ and Hisayoshi Ishii ${ }^{*}$ (1)
}

\begin{abstract}
The skin temperature $\left(T_{m}\right)$ of the orofacial area influences orofacial functions and is related to the blood flow (BF). Marked increases in BF mediated by parasympathetic vasodilation may be important for orofacial $T_{m}$ regulation. Therefore, we examined the relationship between parasympathetic reflex vasodilation and orofacial $T_{m}$ in anesthetized rats. Electrical stimulation of the central cut end of the lingual nerve (LN) elicited significant increases in BF and $T_{m}$ in the lower lip. These increases were significantly reduced by hexamethonium, but not atropine. VIP agonist increased both BF and $T_{m}$ in the lower lip. The activation of the superior cervical sympathetic trunk (CST) decreased BF and $T_{m}$ in the lower lip; however, these decreases were significantly inhibited by LN stimulation. Our results suggest that parasympathetic vasodilation plays an important role in the maintaining the hemodynamics and $T_{m}$ in the orofacial area, and that VIP may be involved in this response.
\end{abstract}

Keywords: Parasympathetic reflex vasodilation, Lingual nerve, Superior cervical sympathetic trunk, VIP, Skin temperature

\section{Introduction}

Local temperature $\left(T_{\mathrm{m}}\right)$ in the orofacial area is generally considered to be important for the maintenance of orofacial functions such as oral sensations $[1,2]$ and wound healing [3]; abnormalities in $T_{\mathrm{m}}$ may be related to orofacial dysfunctions [4-6]. Blood flow (BF), regulated by the autonomic nervous system, in particular, the sympathetic vasoconstrictor fibers that secrete noradrenaline, plays a major role in the regulation of the $T_{\mathrm{m}}$ in the skin of the trunk and limbs $[5,7,8]$. However, the role of the autonomic nervous system in the regulation of $T_{\mathrm{m}}$ in the orofacial area remains unclear.

Two major vasomotor fibers consisting of parasympathetic vasodilator and sympathetic vasoconstrictor fibers

\footnotetext{
${ }^{*}$ Correspondence: hisayosh@hoku-iryo-u.ac.jp

2 Division of Physiology, Department of Oral Biology, School of Dentistry, Health Sciences University of Hokkaido, 1757 Kanazawa, Ishikari-Tobetsu, Hokkaido 061-0293, Japan

Full list of author information is available at the end of the article
}

are located in the orofacial area [9-11]. Parasympathetic vasodilator fibers have been demonstrated to originate from the pterygopalatine, otic, and submandibular ganglia in the orofacial area, and these fibers include acetylcholine and non-cholinergic neurotransmitters such as vasoactive intestinal polypeptide (VIP), as reported in physiological, pharmacological, and histochemical studies [12-15]. Previously, the activation of parasympathetic vasodilator fibers has been reported to occur through trigeminal afferent inputs leading to a rapid and marked increase in BF in orofacial tissues, such as lower lip [16], jaw muscles $[17,18]$ and salivary glands $[15,19]$. On the other hand, sympathetic vasoconstriction is under tonic control from the superior cervical sympathetic trunk (CST) [20-22]. Therefore, parasympathetic vasodilation mediated through the trigeminal reflex mechanisms and interaction between parasympathetic and sympathetic fibers may play an important role in the regulation of both hemodynamics and $T_{\mathrm{m}}$ in the orofacial area. 
However, to the best our knowledge, the relationship between autonomic vasomotor responses and $T_{\mathrm{m}}$ in the orofacial tissues during trigeminal afferent input has not been evaluated so far.

In the present study, we explored the effects of parasympathetic vasodilation evoked by the trigeminal-mediated reflex and sympathetic vasoconstriction (induced by the CST). In addition, the underlying mechanisms mediating these responses and their interactions on $\mathrm{BF}$ and $T_{\mathrm{m}}$ in the lower lip were examined using deeply urethane-anesthetized, artificially ventilated, vagotomized, and sympathectomized rats (Fig. 1).

\section{Methods}

\section{Preparation of animals}

The experiments were performed on 42 adult male Wistar rats (9-15 weeks of age, weighing 295-480 g). After induction of anesthesia using isoflurane, urethane $(1 \mathrm{~g} / \mathrm{kg}$ in a volume of $1 \mathrm{ml} / 100 \mathrm{~g}$ body weight) was injected subcutaneously into the backs of the animals. Room temperature was maintained at $25 \pm 1{ }^{\circ} \mathrm{C}$ during the experiments. One femoral vein was cannulated to allow for the drug injection, and a femoral artery was cannulated and connected to a Statham pressure transducer to monitor the systemic arterial blood pressure (SABP) and heart rate (HR). The anesthetized animals were intubated, paralyzed by intravenous (iv) injection of pancuronium bromide (Mioblock; Organon, Teknika, the Netherlands; $0.6 \mathrm{mg} / \mathrm{kg}$ initially, supplemented with $0.4 \mathrm{mg} / \mathrm{kg}$ every hour or so after testing the level of anesthesia; see below), and artificially ventilated via a tracheal cannula with a mixture of $50 \%$ air and $50 \% \mathrm{O}_{2}$. The ventilator (model SN-480-7; Shinano, Tokyo, Japan) was set to deliver a tidal volume of $8.5-10 \mathrm{~cm}^{3} / \mathrm{kg}$ at a rate of 20-23 breaths/min, and the end-tidal concentration of $\mathrm{CO}_{2}$ was determined by means of an infrared analyzer (Capnomac Ultima; Datex, Helsinki, Finland), as reported elsewhere $[15,17,22]$. This method of continuous ventilation has been shown to maintain the end-tidal concentration of $\mathrm{CO}_{2}$ at $40-45 \mathrm{mmHg}$. The changes in the end-tidal $\mathrm{CO}_{2}$ concentration following each treatment (from 45 to $35 \mathrm{mmHg}$ ) were independent

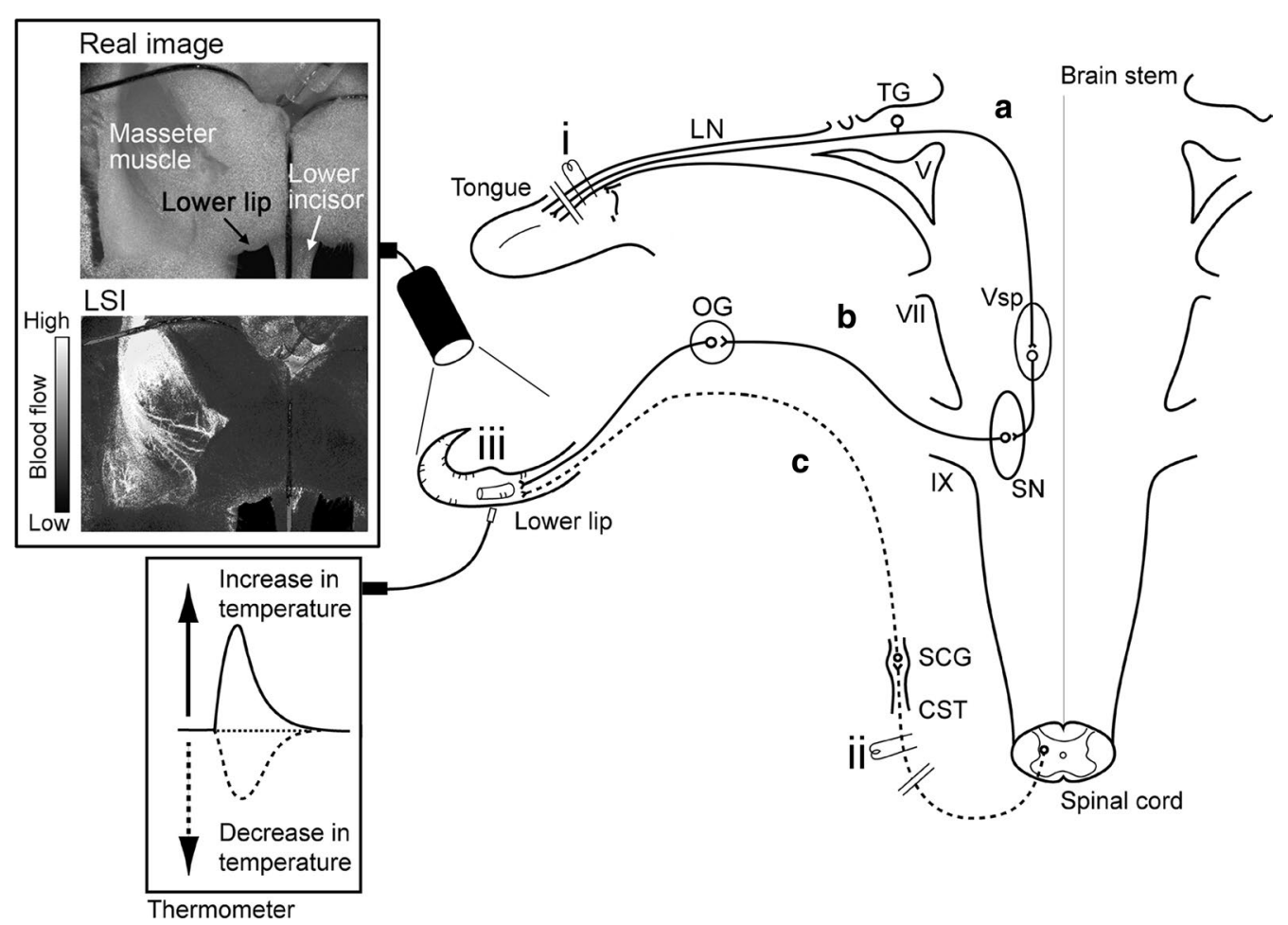

Fig. 1 Schematic representation of the electrical stimulation sites and measurement sites of both local blood flow $(B F)$ and temperature $\left(T_{m}\right)$ in rats. The stimulation sites were as follows: (i) central cut end of the lingual nerve (LN) and (ii) peripheral cut end of the superior cervical sympathetic trunk (CST). Local BF and $T_{m}$ were measured in the (iii) lower lip (Real image), using laser speckle imaging (LSI) and a thermometer, respectively. The continuous lines indicate $\mathbf{a}$ trigeminal sensory inputs to the trigeminal spinal nucleus $\left(\mathrm{V}_{\mathrm{Sp}}\right)$ in the brainstem and $\mathbf{b}$ parasympathetic vasodilator fiber to the lower lip from the salivatory nuclei (SN). The dashed lines indicate sympathetic vasoconstrictor fiber to the lower lip from the superior cervical ganglion (SCG) of the superior CST (c). OG otic ganglion, TG trigeminal ganglion, V trigeminal nerve root, VII facial nerve root, IX glossopharyngeal nerve root. Modified from Ishii et al. [17] 
of the changes in the $\mathrm{BF}$ and $T_{\mathrm{m}}$ measured by the present method (data not shown). Rectal temperature was maintained at $37-38{ }^{\circ} \mathrm{C}$ using a heating pad. Before the injection of additional pancuronium bromide, the adequacy of the depth of anesthesia was determined by the absence of the flexion response to a noxious stimulus, such as pinching the digit for approximately $2 \mathrm{~s}$. The criterion for the maintenance of an adequate depth of anesthesia following paralysis was the absence of a reflex elevation of the $\mathrm{SABP}$ in response to a noxious stimulus. When the depth of anesthesia was considered inadequate, additional urethane (intermittent doses of $100 \mathrm{mg} / \mathrm{kg}$, iv) was administered. At the end of the experiment, all rats were killed by an overdose (approximately $100 \mathrm{mg}$, iv) of pentobarbital sodium. The experimental protocols were reviewed and approved by the Animal Ethics and Research Committee and conducted in accordance with the Regulations for the Care and Use of Laboratory Animals of the Health Sciences University of Hokkaido (No. 075). All the animals were cared for in accordance with the recommendations in the current National Research Council guide.

\section{Measurement of cardiovascular parameters and local $T_{\mathbf{m}}$ in the orofacial area}

Changes in BF in the lower lip (Fig. 1, iii) and skin of the dorsum of the foot on the left side were monitored using a laser speckle flowmeter (Omegazone; Omegawave, Tokyo, Japan), which obtains the high-resolution twodimensional images in seconds, as described previously $[15,23,24]$. A $780-\mathrm{nm}$ semiconductor laser was used to illuminate the surface of the orofacial area. The scattered light was filtered and detected by a charge-coupled device $(C C D)$ camera positioned above the measuring sites. Raw speckle images (real images) corresponding to the number and velocity of moving red blood cells (BF) were collected by the CCD camera and transferred to a computer for analysis. Color-coded BF images (speckle images) were obtained in high-resolution mode (638 pixels $\times 480$ pixels; 1 image/s). One BF image was generated by averaging the numbers obtained from 20 consecutive raw speckle images. The averaged signals in $\mathrm{BF}$ at the regions of interest (ROI), which indicated the highest increases in BF, were obtained using the pallet software installed in the Omegazone imaging system (Omegawave, Tokyo, Japan). The analog output of the equipment did not provide absolute values but demonstrated the relative changes in BF expressed in arbitrary units (a.u.) [25]. The SABP was recorded from a femoral catheter via a Statham pressure transducer. The HR, as well as the systolic, diastolic, and mean SABP were calculated from the SABP signals $(n=7$, Table 1$)$. Vascular conductance (VC) was calculated using the following equation:

$$
\mathrm{VC}(\text { a.u./mmHg })=\mathrm{BF} \text { (a.u.)/SABP }(\mathrm{mmHg})
$$

Local $T_{\mathrm{m}}$ was measured using a non-contact thermometer (PT-3S, OPTEX, Shiga, Japan) (Fig. 1), which measures the surface $T_{\mathrm{m}}$ of objects by caching the infrared energy emitted by the target objects $(2.5 \mathrm{~mm}$ diameter). All data were collected online using a LabScribe2 dataacquisition system (iWorx systems, Washington, $\mathrm{NH}$, USA). Changes $(\Delta)$ in the parameters were assessed by measuring the heights of the maximum values from the baseline in the responses (Fig. 2) unless otherwise noted.

\section{Electrical stimulation of the $\mathrm{LN}$ and superior CST}

The central cut end of the lingual nerve (LN; Fig. 1, i) and the peripheral cut end of the superior CST (Fig. 1, ii) were electrically stimulated using a bipolar silver electrode attached to an electrical stimulator (model SEN7103; Nihon Kohden, Tokyo, Japan). For this purpose, the nerves were stimulated unilaterally under a binocular microscope. The LN was stimulated for $20 \mathrm{~s}$ using a supramaximal voltage $(20 \mathrm{~V})$ at various frequencies $(1-20 \mathrm{~Hz})$ for $2-\mathrm{ms}$ pulse duration $[15,17,24]$, either alone or in combination with CST stimulation $(n=6$ in each group). Electrical stimulation of the CST was performed for periods of 2 min using a supramaximal voltage $(10 \mathrm{~V})$ and $2-\mathrm{ms}$ pulse duration at various frequencies $(0.5-5 \mathrm{~Hz})$ [21]. These intensities in both the LN and CST have been reported to be optimal for inducing BF

Table 1 Heart rate and systemic blood pressure responses associated each condition

\begin{tabular}{|c|c|c|c|c|}
\hline $\begin{array}{l}\text { Heart and blood pressure } \\
\text { measurements }\end{array}$ & Baseline & LN simulation & CST simulation & $\mathrm{CST}+\mathrm{LN}$ simulation \\
\hline HR (beats/min) & $422 \pm 23$ & $435 \pm 22$ & $426 \pm 13$ & $396 \pm 7$ \\
\hline Systolic SABP (mmHg) & $121.9 \pm 4.4$ & $166.5 \pm 8.6^{* *}$ & $134.3 \pm 8.2$ & $178.4 \pm 18.2^{*}$ \\
\hline Diastolic SABP (mmHg) & $71.1 \pm 6.1$ & $108.2 \pm 9.3^{* *}$ & $84.1 \pm 8.5$ & $131.1 \pm 16.8^{*}$ \\
\hline Mean SABP (mmHg) & $88.1 \pm 5.4$ & $127.6 \pm 8.9^{* *}$ & $100.8 \pm 8.4$ & $146.9 \pm 17.1^{*}$ \\
\hline
\end{tabular}

Values in table are given as the mean \pm standard error of the mean (SEM) $(n=7)$

$L N$ lingual nerve, $C S T$ cervical sympathetic trunk, $H R$ heart rate, $S A B P$ systemic arterial blood pressure

Significant difference from baseline at ${ }^{*} P<0.05,{ }^{* *} P<0.001$ 


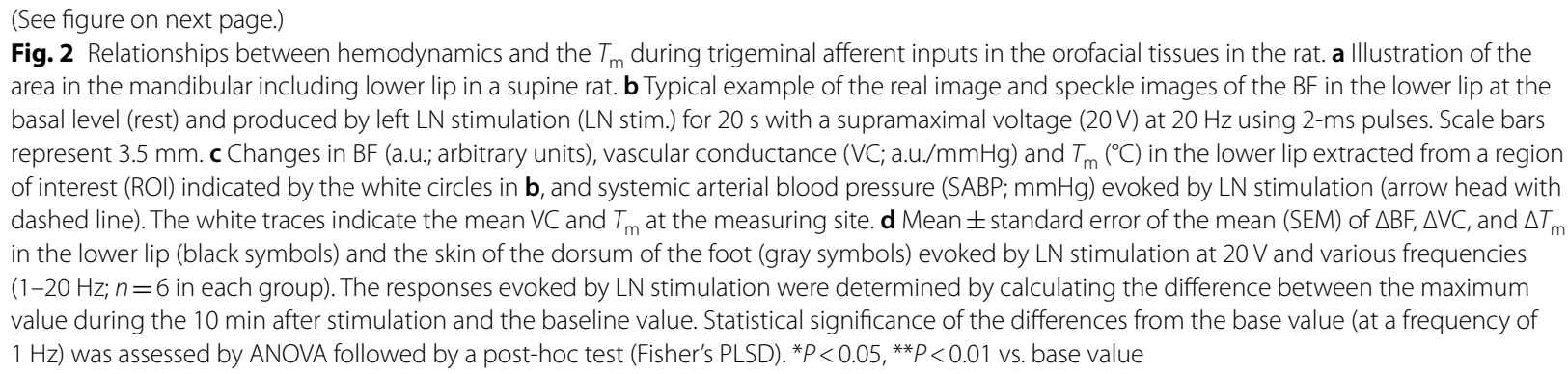

changes via each nerve stimulation as described in our previous studies $[17,21]$. The period of LN stimulation chosen for the present study was $20 \mathrm{~s}$ because the parasympathetic vasodilator fibers were rapidly activated through the trigeminal-mediated reflex, as reported previously [17]. On the other hand, CST stimulation for 2 min appears to mimic the physiological forms of spontaneous tonic activity in the CST fibers supplying the orofacial vasculature. This is because vasoconstriction in the lower lip induced by CST stimulation reached stable levels within $1 \mathrm{~min}$ and sustained these levels during the stimulation (Figs. 5, 6) [21]. In all experiments, the cervical vagi and superior CST were bilaterally transected in the neck, before the stimulation to ensure that only nonvagal parasympathetic effects were examined.

\section{Pharmacological agents}

All drugs were dissolved in sterile saline. The AGP-8633 $(n=6 ; 0.05-5 \mu \mathrm{g} / \mathrm{ml}$, ANYGEN, Korea) was used as a VIP agonist. The following pharmacological interventions were performed: autonomic ganglion cholinergic blockade using hexamethonium bromide $(n=6 ; 10 \mathrm{mg} /$ $\mathrm{ml}$; Sigma-Aldrich, St. Louis, MO) and muscarinic cholinergic blockade using atropine sulfate $(n=6 ; 100 \mu \mathrm{g} /$ ml; Mitsubishi Tanabe, Osaka, Japan). These drugs were perfused intravenously for $10 \mathrm{~min}$ at a flow rate of $0.1 \mathrm{ml} /$ min using a syringe pump (Model '22' Multisyringe; HARVARD, Holliston, MA). The administration of a similar volume of saline alone had no measurable effect on the cardiovascular parameters and local $T_{\mathrm{m}}$ (data not shown). The responses evoked by electrical stimulations after the administration of each drug were determined at least 10 min after injection because changes in $\mathrm{BF}$ and SABP reached a steady-state during this period. The magnitude of the response obtained following the administration of each blocking agent was expressed as a percentage of the control response recorded prior to its administration. The dose of hexamethonium chosen for the present study was $10 \mathrm{mg} / \mathrm{ml}$; a similar dose markedly inhibited the increase in BF in the orofacial area, which was evoked by the activation of the parasympathetic vasodilator fibers through the trigeminal-mediated reflex
$[15,17,24]$. The efficacy of the blockade using atropine was assessed by the absence of a vasodilator response in response to acetylcholine bromide $(100 \mathrm{ng} / \mathrm{kg}$, iv; SigmaAldrich, St. Louis, MO).

\section{Statistical analysis}

All numerical data are presented as means \pm standard error of the mean (SEM). The statistical significance of observed changes was assessed using paired Student's $t$ test or analysis of variance (ANOVA) followed by a posthoc test [Fisher's protected least significant difference (PLSD) test]. Differences in means were considered significant at $P<0.05$. Data were analyzed using a Macintosh computer with StatView 5.0 (SAS Institute Inc., Cary, $\mathrm{NC}$.

\section{Results}

\section{Effects of electrical stimulation of the central cut end} of the LN on the hemodynamics and local $T_{m}$ in the lower lip, and SABP

Figure 2 shows the changes in the $\mathrm{BF}, \mathrm{VC}$, and $T_{\mathrm{m}}$ of the lower lip, skin of the dorsum of the foot, and SABP before and after electrical stimulation of the central cut end of $\mathrm{LN}$ on the left side. The basal $\mathrm{BF}, \mathrm{VC}$, and $T_{\mathrm{m}}$ levels in the lower lip were $3.5 \pm 0.3$ a.u., $0.05 \pm 0.01$ a.u. $/ \mathrm{mmHg}$, and $33.4 \pm 0.6{ }^{\circ} \mathrm{C}$, respectively. Electrical stimulation of the left $\mathrm{LN}$ for $20 \mathrm{~s}$ with $20 \mathrm{~V}$ and $2-\mathrm{ms}$ pulses at $20 \mathrm{~Hz}$ increased $\mathrm{BF}, \mathrm{VC}$, and $T_{\mathrm{m}}$ in the lower lip on the left side, but not on the right side (Fig. 2b). Frequency-response curves were generated using stimulus trains $(1-20 \mathrm{~Hz})$ at $20 \mathrm{~V}$ (Fig. 2d). Significant changes in $\triangle B F, \triangle V C$, and $\Delta T_{\mathrm{m}}$ evoked by LN stimulation in the lower lip occurred at frequencies above $5 \mathrm{~Hz}$ (for $\Delta \mathrm{BF}, F_{4,25}=5.05, P<0.01$; for $\Delta \mathrm{VC}, F_{4,25}=2.87, P<0.05$; for $\Delta T_{\mathrm{m}}, F_{4,25}=4.99$, $P<0.01)$. In the contrast, electrical stimulation of the LN failed to affect the values in the skin of the dorsum of the foot (Fig. 2d). The animals exhibited normal systolic and diastolic pressures, mean SABP, and HR during rest (Table 1). The HR remained unchanged during LN stimulation $(20 \mathrm{~V}, 20 \mathrm{~Hz}, 20 \mathrm{~s})$ (NS, paired $t$ test). However, significant differences in the SABP before and after LN stimulation were noted $(P<0.001$, paired $t$ test) (Table 1$)$. 

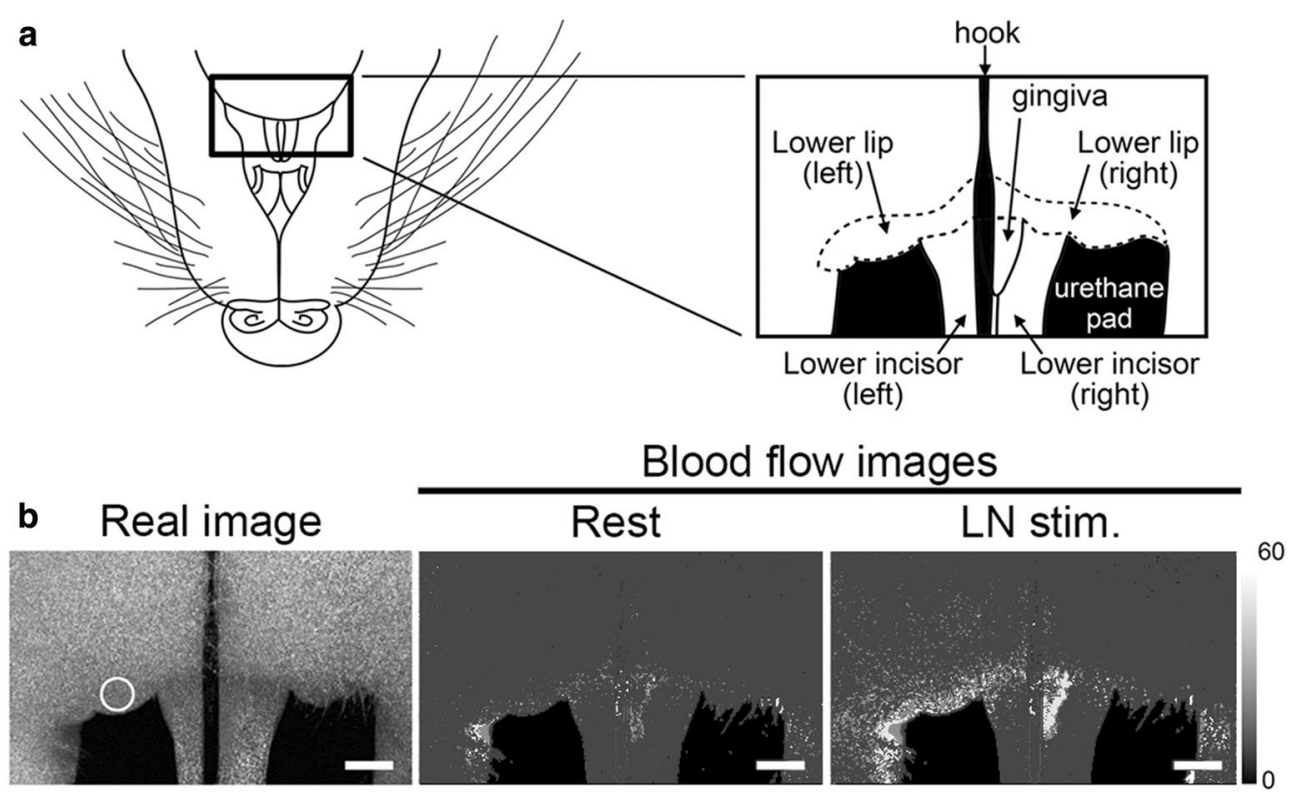

C
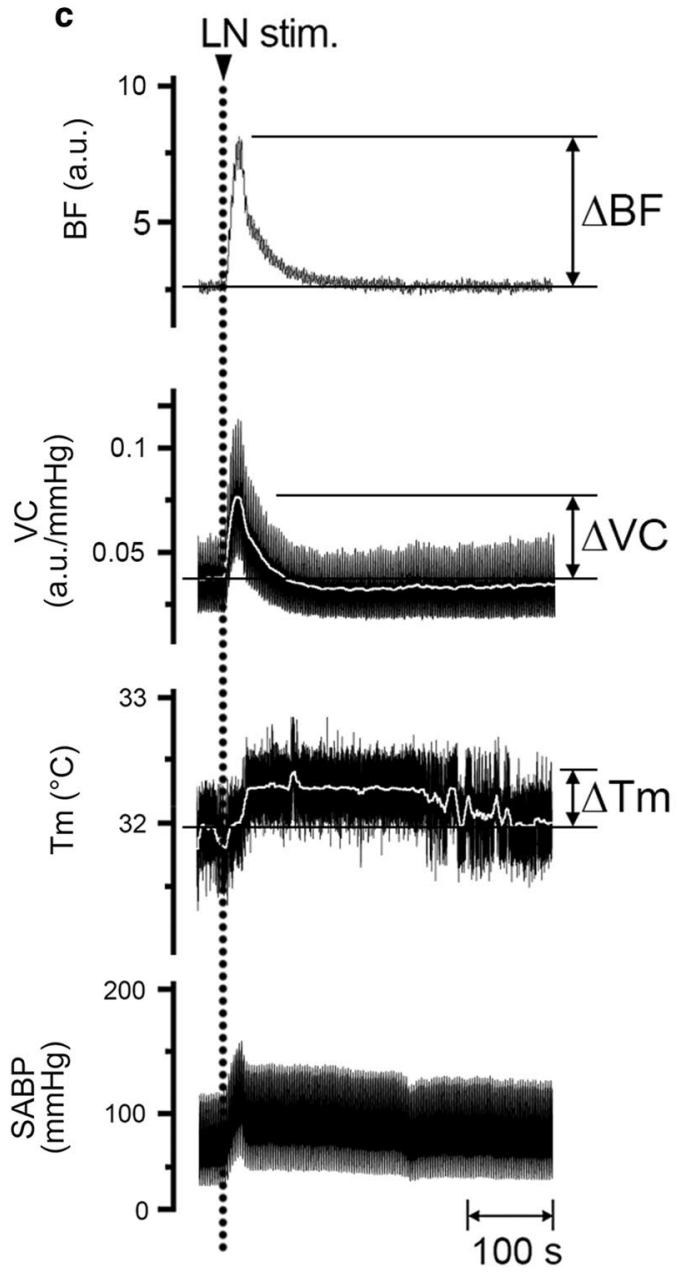

d
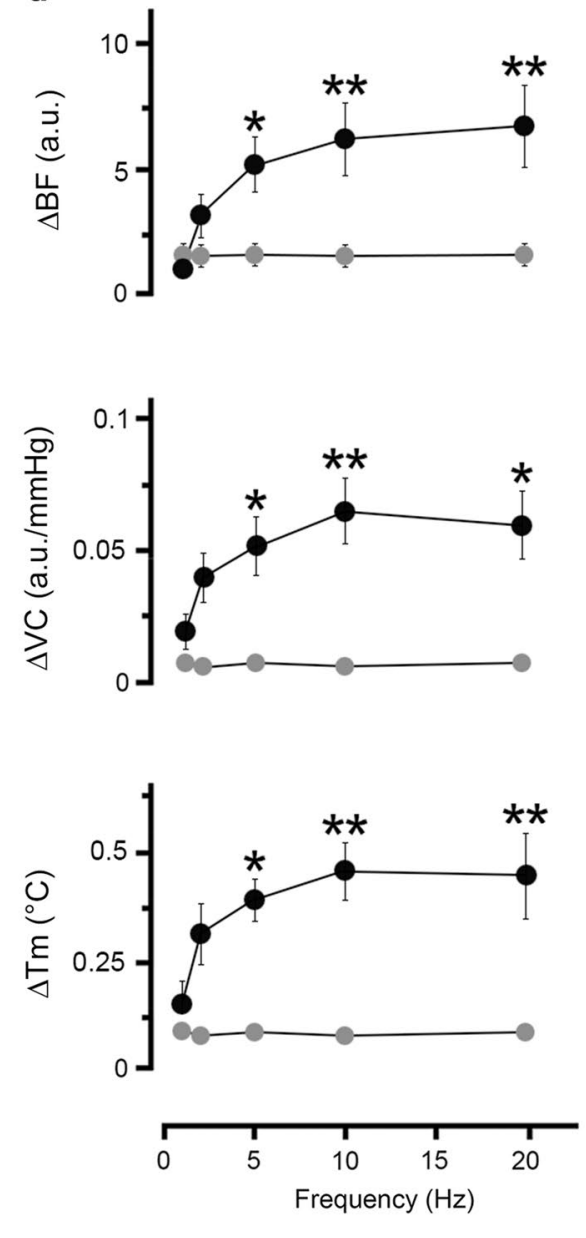
Effects of pharmacological blocking agents on the increase in the $\mathrm{BF}$ and $T_{m}$ in the lower lip evoked by LN stimulation Increases in $\mathrm{BF}, \mathrm{VC}$, and $T_{\mathrm{m}}$ in the lower lip on the left side evoked by left LN stimulation $(20 \mathrm{~s}, 20 \mathrm{~V}, 20 \mathrm{~Hz}$, 2-ms) were almost abolished by the intravenous administration of hexamethonium $\left(\mathrm{C}_{6}, 10 \mathrm{mg} / \mathrm{ml}\right)$ (Fig. 3a). Significant differences in the $\Delta \mathrm{BF}, \Delta \mathrm{VC}$, and $\Delta T_{\mathrm{m}}$ evoked by LN stimulation in the lower lip before and after hexamethonium administration were observed (for $\Delta \mathrm{BF}, F_{2,15}=47.5, P<0.001$; for $\Delta \mathrm{VC}, F_{2,15}=19.6$, $P<0.001$; for $\Delta T_{\mathrm{m}}, F_{2,15}=24.9, P<0.001$, ANOVA followed by Fisher's PLSD; Fig. 3c). The responses returned close to the initial value at 30-60 min after hexamethonium administration (data not shown). Administration of atropine $(100 \mu \mathrm{g} / \mathrm{ml})$ had no effect on the response (Fig. 3b, c). The HR values at $10 \mathrm{~min}$ after the administration of hexamethonium and atropine were $371 \pm 19$ and $400 \pm 5$ beats/min, respectively, and mean SABP values 10 min after the administration of hexamethonium and atropine were $60.5 \pm 2.9 \mathrm{mmHg}$ and $108.7 \pm 24.6 \mathrm{mmHg}$, respectively. Statistically significant differences in the mean SABP before and after
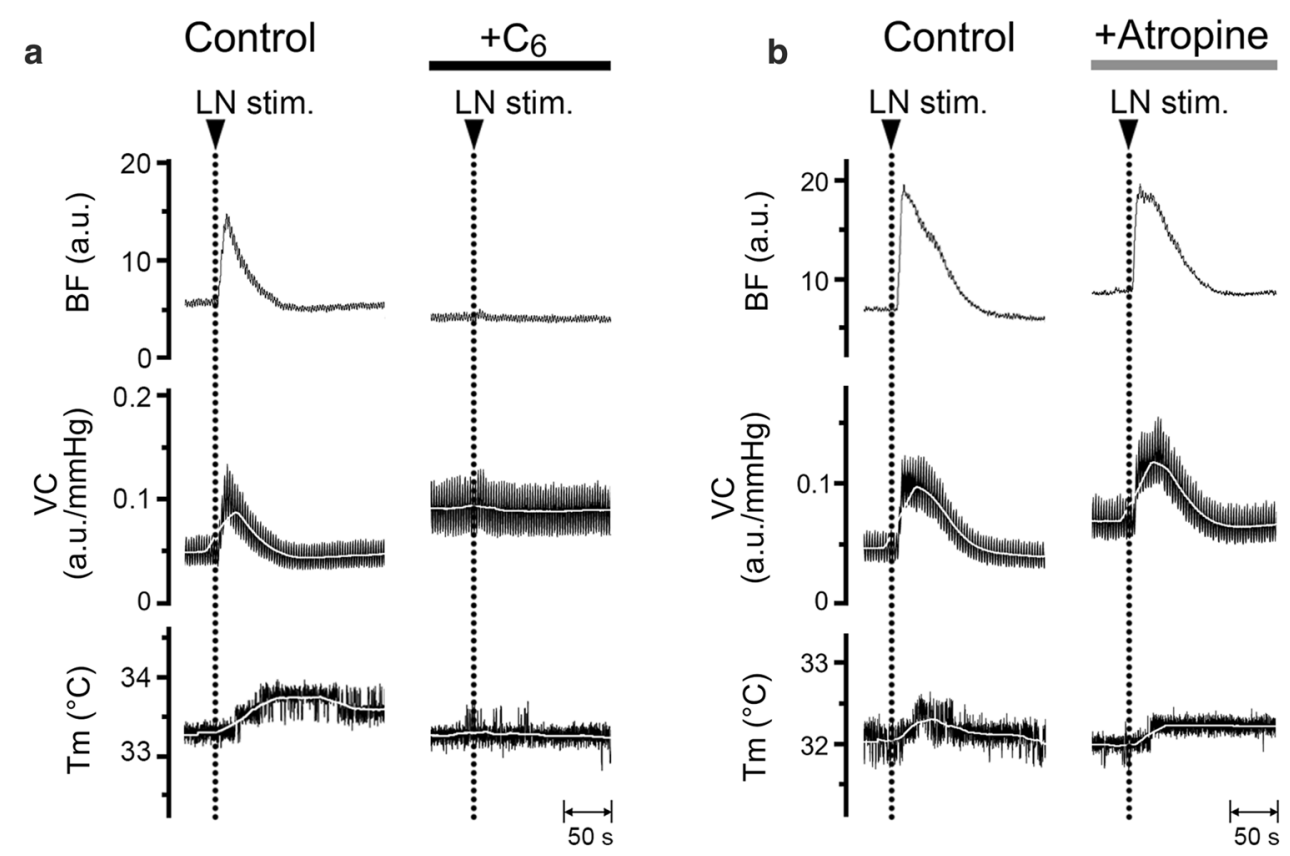

$\mathbf{C}$

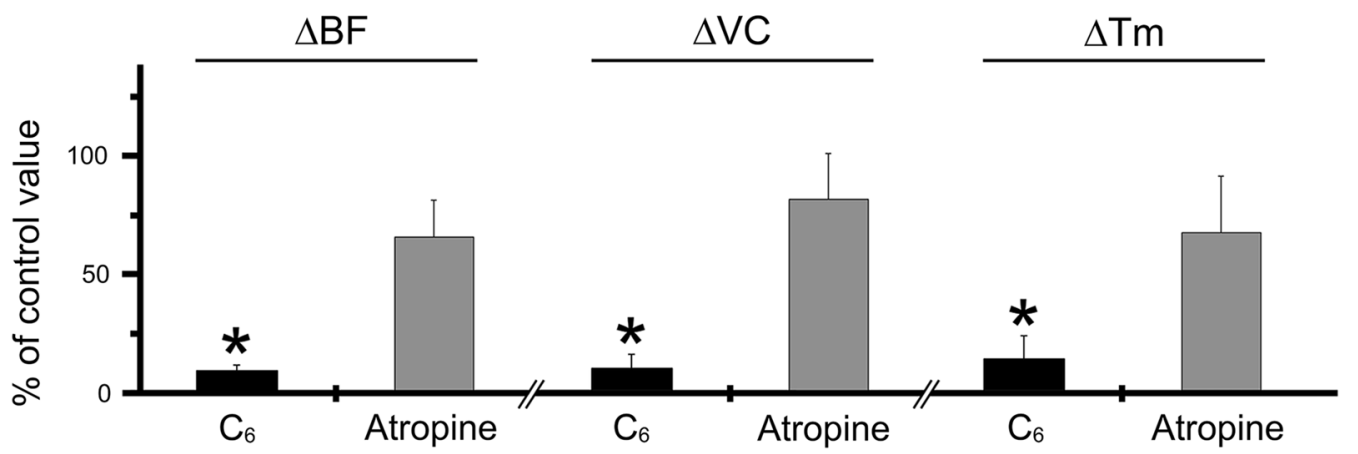

Fig. 3 Effects of pharmacological blocking agents on the increase in BF and $T_{m}$ evoked by LN stimulation in the lower lip. Typical examples of the effects of intravenous administration of hexamethonium $\left(C_{6}\right)$ at $10 \mathrm{mg} / \mathrm{ml}(\mathbf{a})$ and atropine $(\mathbf{b})$ at $100 \mu \mathrm{g} / \mathrm{ml}$ for $10 \mathrm{~min}(0.1 \mathrm{ml} / \mathrm{min})$ on changes in the $B F, V C$, and $T_{m}$ in the lower lip on the left side evoked by left $L N$ stimulation $(20 \mathrm{~s}, 10 \mathrm{~V}, 20 \mathrm{~Hz}, 2-\mathrm{ms})$. c Mean $\pm \mathrm{SEM}$ of changes in the $\triangle B F, \triangle V C$, and $\Delta T_{m}$ in the lower lip evoked by $L N$ stimulation with administration of $C_{6}$ (black bars) and atropine (gray bars; $n=6$ in each group). The responses evoked by $L N$ stimulation with $C_{6}$ and atropine were determined by calculating the differences between the maximum values during 10 min after stimulation with $C_{6}$ and atropine and the baseline values. Each value is expressed as a percentage of the response before treatment (control). Statistical significance was assessed by ANOVA followed by a post-hoc test (Fisher's PLSD). ${ }^{*} P<0.001$ vs. control 
the administration of hexamethonium $(P<0.001)$, but not atropine, were noted.

\section{Effects of exogenously applied VIP agonist}

\section{on the hemodynamics and $T_{\mathrm{m}}$ in the lower lip}

Figure 4a shows the effects of intravenous administration of a VIP agonist at $(5 \mu \mathrm{g} / \mathrm{ml})$ on the $\mathrm{BF}, \mathrm{VC}$, and $T_{\mathrm{m}}$ in the lower lip on the left side. The administration of the VIP agonist induced increases in BF, VC, and $T_{\mathrm{m}}$ in the lower lip in a dose-dependent manner $(0.05-5 \mu \mathrm{g} /$ $\mathrm{ml}$; Fig. $4 \mathrm{~b})$. Significant changes in $\triangle \mathrm{BF}$ and $\triangle \mathrm{VC}$ were evoked by the agonist at $5 \mu \mathrm{g} / \mathrm{ml}$; likewise, the changes in $\Delta T_{\mathrm{m}}$ were evoked by the agonist at dose above $0.5 \mu \mathrm{g} /$ $\mathrm{ml}$ (for $\Delta \mathrm{BF}, F_{2,15}=5.78, P<0.05$; for $\Delta \mathrm{VC}, F_{2,15}=8.59$, $P<0.01$; for $\Delta T_{\mathrm{m}}, F_{2,15}=7.14, P<0.01$; Fig. $\left.4 \mathrm{~b}\right)$. The HR at $10 \mathrm{~min}$ after each dose of VIP was $410 \pm 17$ beats $/ \mathrm{min}$. No statistically significant differences in the HR before and after its administration were observed. The mean SABP 10 min after VIP agonist administration at dose of $0.05,0.5$, and $5 \mu \mathrm{g} / \mathrm{ml}$ were $113.7 \pm 5.2,111.9 \pm 5.1$, and $76.1 \pm 5.2 \mathrm{mmHg}$, respectively. A significant difference in mean SABP before and after the administration of VIP agonist at $5 \mu \mathrm{g} / \mathrm{ml}$ was observed $(P<0.001$, paired $t$ test).
Effects of electrical stimulation of the peripheral cut end of the CST on the hemodynamics and $T_{m}$ in the lower lip, and the SABP

Figure 5 shows the changes in the $\mathrm{BF}, \mathrm{VC}$, and $T_{\mathrm{m}}$ in the lower lip on the left side, and in SABP before and after electrical stimulation of the peripheral cut end of the left CST. Electrical stimulation for 2 min with $10 \mathrm{~V}$ at $5 \mathrm{~Hz}$ using 2-ms pulses of the left CST decreased BF, VC, and $T_{\mathrm{m}}$ in the lower lip (Fig. 5a). Frequencyresponse curves were generated using stimulus trains $(0.5-5 \mathrm{~Hz})$ at $10 \mathrm{~V}$ (Fig. $5 \mathrm{~b})$. Significant changes in $\Delta \mathrm{BF}$, $\Delta T_{\mathrm{m}}$, and $\Delta \mathrm{VC}$ evoked by CST stimulation in the lower lip occurred at frequencies above 1 and $2 \mathrm{~Hz}$ (for $\Delta \mathrm{BF}$, $F_{3,20}=3.18, P<0.05$; for $\Delta \mathrm{VC}, F_{3,20}=3.39, P<0.05$; for $\left.\Delta T_{\mathrm{m}}, F_{3,20}=6.21, P<0.01\right)$. The HR and SABP remained unchanged during CST stimulation (Table 1). No significant differences in HR and mean SABP were observed before and after CST stimulation.

\section{Effects of CST stimulation in combination with LN stimulation on the hemodynamics and $T_{\mathrm{m}}$ in the lower lip, and SABP}

Figure 6 shows the effects of CST stimulation $(2 \mathrm{~min}$, $10 \mathrm{~V}, 5 \mathrm{~Hz}, 2-\mathrm{ms}$ ) alone (control) and in combination
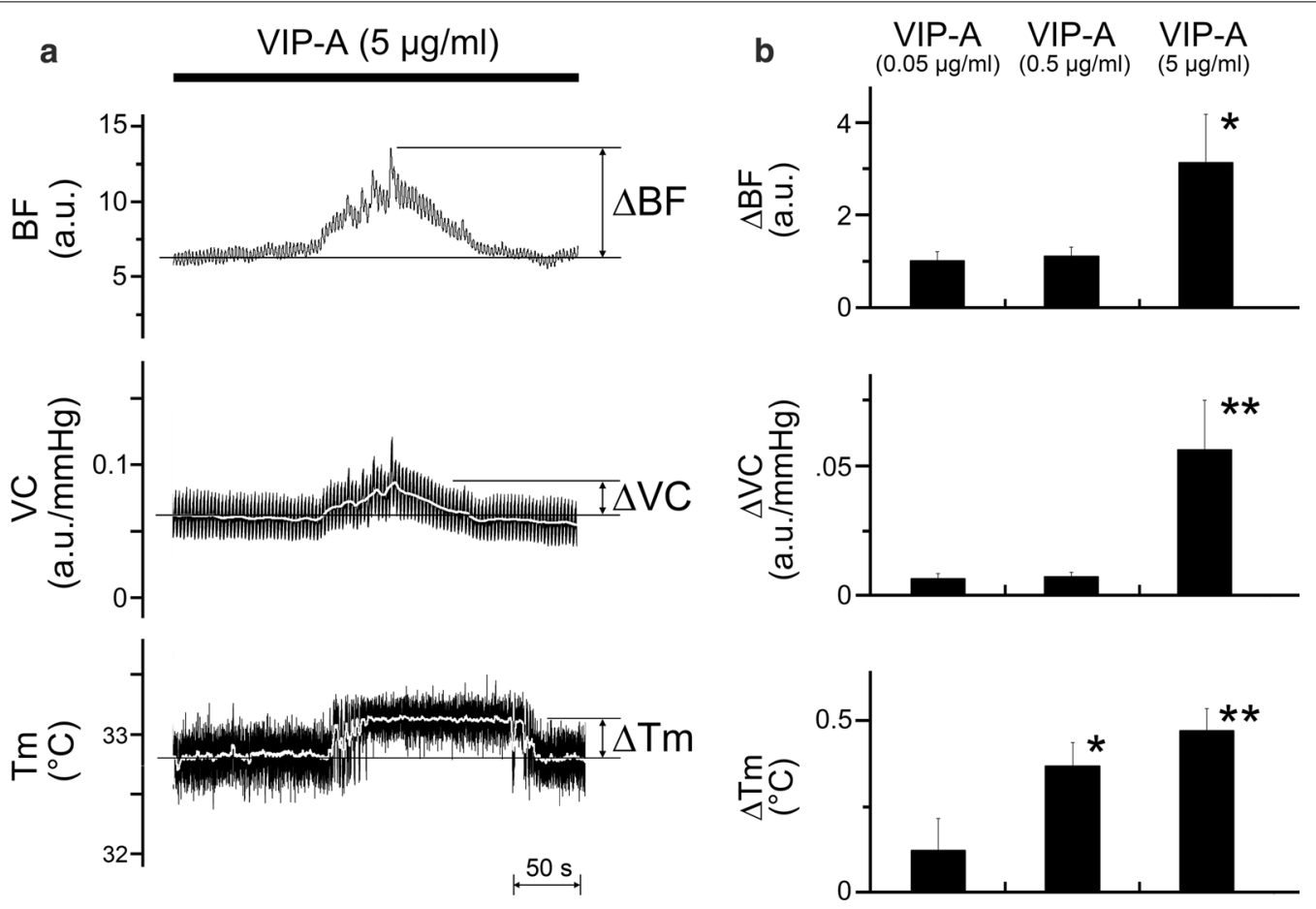

Fig. 4 Effects of an exogenously applied VIP agonist on the hemodynamics and $T_{m}$ in the lower lip. a Typical examples of the effects of intravenous administration of VIP agonist (VIP-A) at $5 \mu \mathrm{g} / \mathrm{ml}$ for $10 \mathrm{~min}(0.1 \mathrm{ml} / \mathrm{min})$ on changes in $\mathrm{BF}, \mathrm{VC}$, and $T_{\mathrm{m}}$ in the lower lip on the left side. $\mathbf{b}$ Mean \pm SEM of changes in the $\triangle B F, \Delta V C$, and $\Delta T_{m}$ in the lower lip evoked by the drug at $0.05-5 \mu \mathrm{g} / \mathrm{ml}$ ( $n=6$ in each group). The responses evoked by the administration of a VIP agonist were determined by calculating the difference between the maximum value during the 10 min after its administration and the value at baseline. ${ }^{*} P<0.05,{ }^{* *} P<0.01$ vs. base value 

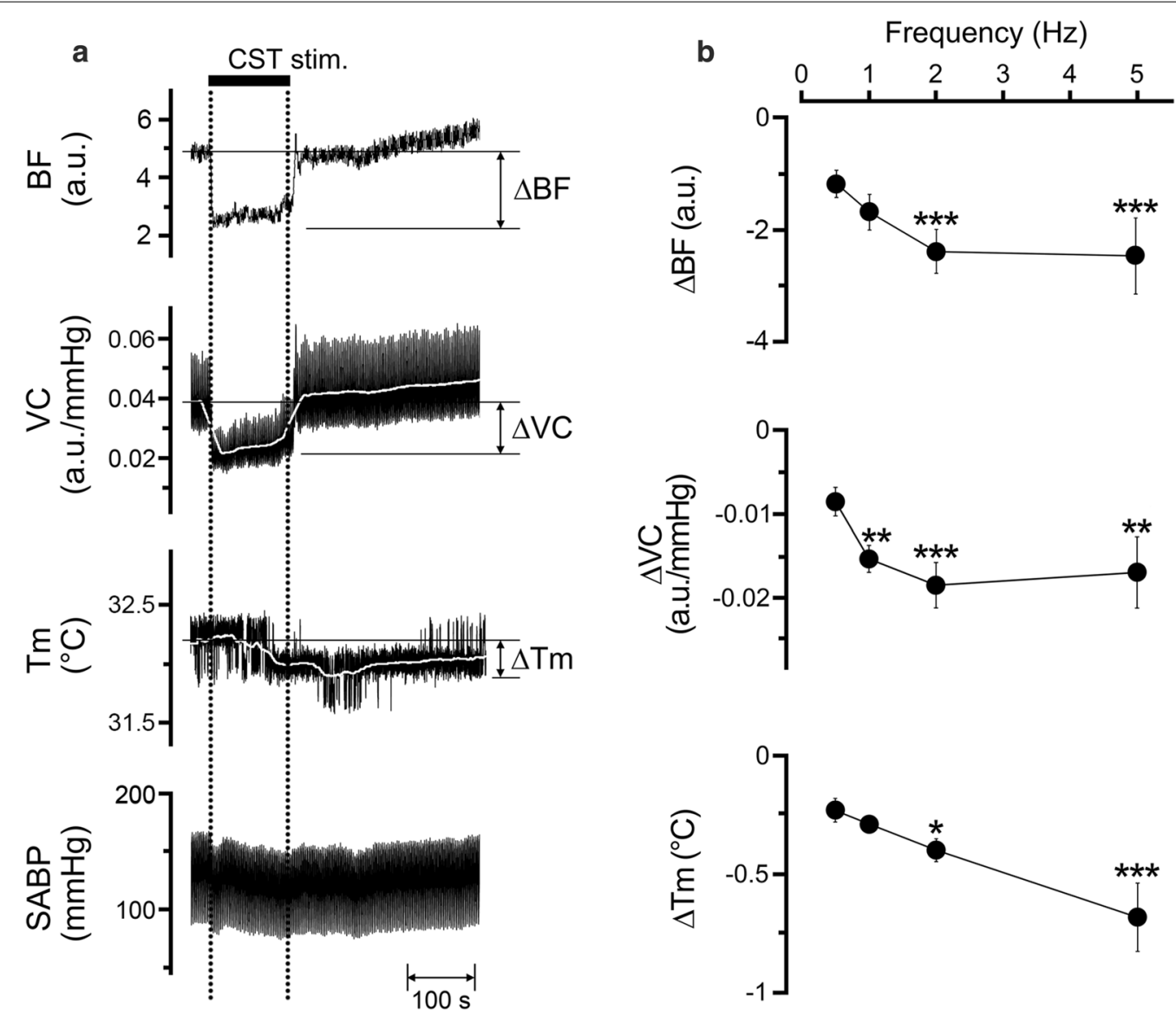

Fig. 5 Sympathetic effects on the hemodynamics and $T_{m}$ in the lower lip, and SABP. a Typical examples of changes in BF, VC, and $T_{m}$ in the lower lip on the left side, extracted from the ROI indicated by the white circles in Fig. 2b, and SABP evoked by electrical stimulation of the peripheral cut end of the left CST (CST stim.; horizontal bar with dashed lines) for 2 min with a supramaximal voltage (10 V) at $5 \mathrm{~Hz}$ using 2-ms pulses. b Mean \pm SEM of $\triangle B F, \triangle V C$, and $\Delta T_{m}$ in the lower lip (black symbols) evoked by CST stimulation at $10 \mathrm{~V}$ and various frequencies $(0.5-5 \mathrm{~Hz} ; n=6$ in each group). The responses evoked by CST stimulation were determined by calculating the difference between the maximum value during the 10 min after stimulation and the baseline value. ${ }^{*} P<0.05,{ }^{* *} P<0.01,{ }^{* * *} P<0.001 \mathrm{vs}$. base value

with LN stimulation (20 s, $20 \mathrm{~V}, 20 \mathrm{~Hz}, 2-\mathrm{ms})$ on the left side. Decreases in BF, VC, and $T_{\mathrm{m}}$ in the lower lip evoked by CST stimulation were inhibited by LN stimulation (Fig. 6a). Furthermore, significant differences in $\Delta \mathrm{BF}(P<0.01), \Delta \mathrm{VC}(P<0.001)$, and $\Delta T_{\mathrm{m}}(P<0.05)$ in the lower lip were observed between CST stimulation alone and in combination with LN stimulation (paired $t$ test; Fig. 6b). HR remained unchanged during each stimulus condition (Table 1). Significant differences in SABP before and after CST stimulation in combination with LN stimulation were observed $(P<0.05$, paired $t$ test; Table 1).

\section{Discussion}

Our results showed that electrical stimulation of the central cut end of the LN in cervically sympathectomized and vagotomized rats significantly increased the BF and $T_{\mathrm{m}}$ in a frequency-dependent manner on the ipsilateral side of the lower lip (Fig. 2). The observed increases in $\mathrm{BF}$ and $T_{\mathrm{m}}$ were found to be unrelated to changes in the SABP due to the following reasons: the $\mathrm{VC}$ in each site was significantly increased by LN stimulation; no significant increases in $\mathrm{BF}$ and $T_{\mathrm{m}}$ were noted on the contralateral side of the lower lip and the skin of the dorsum of the foot (measured simultaneously; Fig. 2); and no significant differences in the HR before and after LN stimulation were observed (Table 1). These results suggest that increases in BF and Tm elicited by $\mathrm{LN}$ stimulation are not a passive result of any evoked SABP or HR changes and that these increases are likely the result of vasodilation. The evocation of vasodilation through the trigeminal afferent may play an important role in the regulation of the hemodynamics and $T_{\mathrm{m}}$ in the orofacial area under physiological conditions. This is because LN stimulationinduced BF increase has been reported in some orofacial tissues including the lower lip, regardless of the presence 

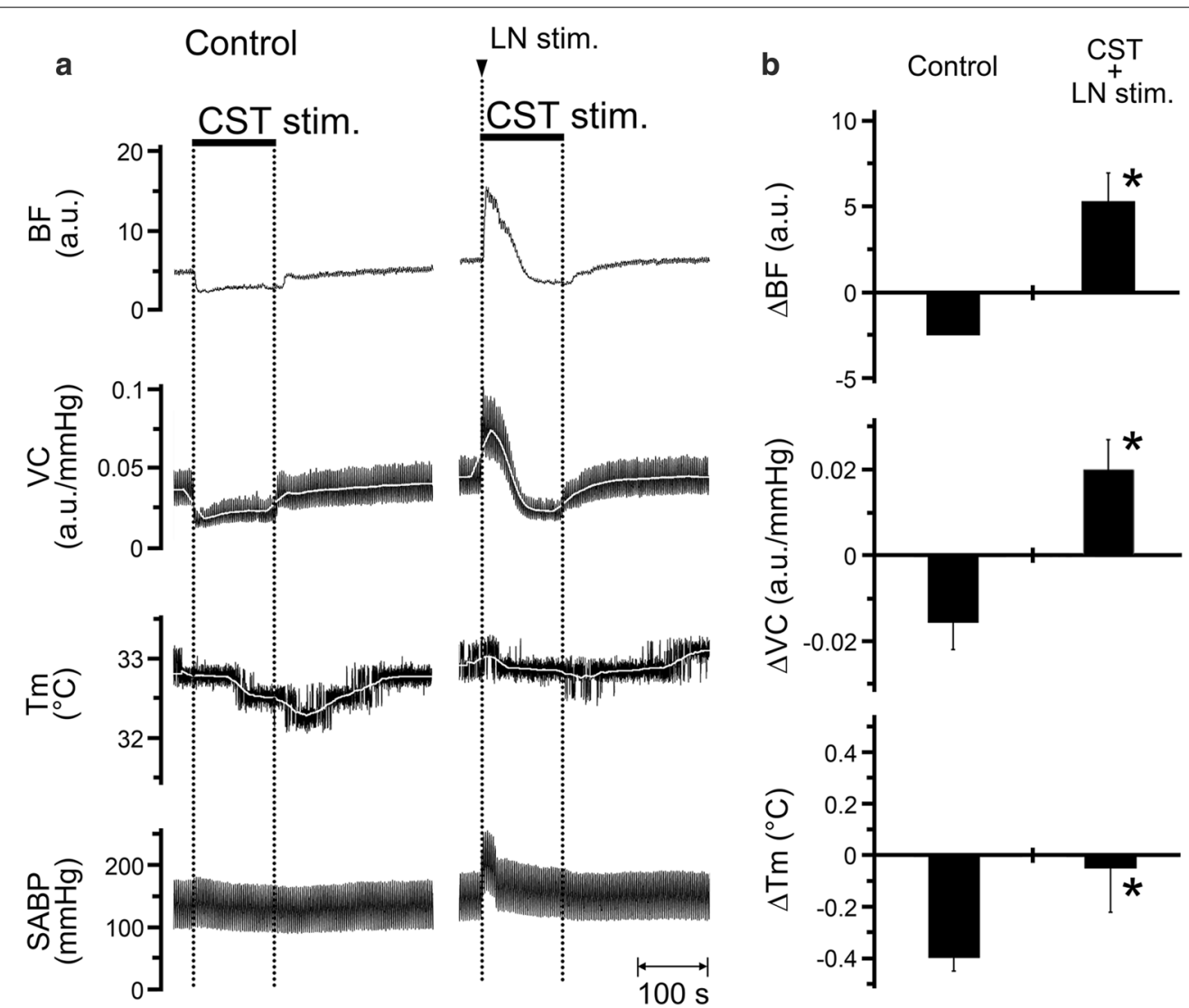

Fig. 6 Interactions between trigeminally mediated parasympathetic vasodilation and sympathetic vasoconstriction in the regulation of the hemodynamics and $T_{m}$ in the orofacial area. a Typical examples of the changes in BF, VC, and $T_{m}$ in the lower lip on the left side, and SABP evoked by left CST stimulation (CST stim.) alone (2 min, 10 V, $5 \mathrm{~Hz}, 2$-ms; control) and in combination with left LN stimulation (20 s, $20 \mathrm{~V}, 20 \mathrm{~Hz}, 2-\mathrm{ms})$. Changes $(\Delta)$ in the parameters were assessed by calculating the sum of the maximum $(+)$ and minimum $(-)$ values from the baseline in the responses. b Mean data $\pm S E M$ of $\triangle B F, \triangle V C$, and $\triangle T_{m}$ in the lower lip (black bars) evoked by left CST stimulation alone (control) and in combination with $L N$ stimulation (CST + LN stim.; $n=6$ in each group). Statistical significance of the differences from control was assessed by paired Student's $t$ test. ${ }^{*} P<0.001$ vs. control

(CST intact) or absence (sectioning of the CST) of the sympathetic innervation [17].

The increase in both $\mathrm{BF}$ and $T_{\mathrm{m}}$ in the lower lip followed by LN stimulation was prominently reduced by the intravenous administration of hexamethonium, whereas the administration of atropine had no significant effect on either response (Fig. 3). These results suggest that $\mathrm{LN}$ stimulation-induced increases in $\mathrm{BF}$ and $T_{\mathrm{m}}$ in the lower lip are mediated by the activation of parasympathetic reflex vasodilation, which is mediated by final neurons via a non-cholinergic response. This is in accord with the observations that LN stimulation-induced BF increase in the rat lower lip is almost mediated through the atropine-resistant parasympathetic vasodilation [17]. The neural mechanisms underlying non-cholinergic parasympathetic vasodilation in the orofacial area are not fully understood. However, this response may be mediated, in part, by VIP because the intravenous administration of a
VIP agonist induced an increase in both BF and $T_{\mathrm{m}}$ in the lower lip in a dose-dependent manner (Fig. 4). This was supported by the findings that VIP immunoreactivity is observed in the otic and submandibular ganglion and in the nerve fibers that innervate the blood vessels in the lip $[12,26]$. Furthermore, the intravenous administration of VIP also induces vasodilation in the masseter muscle [14] and submandibular gland [15], which is markedly suppressed by a selective VIP receptor antagonist. However, further investigations are necessary to establish the neural mechanisms underlying the non-cholinergic parasympathetic vasodilation in the orofacial area.

The increase in BF in the lower lip evoked by LN stimulation was phasic, whereas the increase in $T_{\mathrm{m}}$ appeared to be a longer response. The precise reasons for the differences in the durations of the responses are unclear, but it may be due to the widely induced increase in BF during LN stimulation in the lower lip (Fig. 2). This suggests that 
parasympathetic reflex vasodilation may be involved in maintaining a continuous $T_{\mathrm{m}}$ in the orofacial area.

Electrical stimulation of the peripheral cut end of the CST significantly decreased BF and $T_{\mathrm{m}}$ in a frequencydependent manner in the lower lip, on the ipsilateral side (Fig. 5). The decrease induced by CST stimulation appeared to be vasoconstriction because no significant changes in the SABP and HR were observed during its stimulation (Table 1). This indicates that sympathetic vasoconstriction evoked by excess sympathetic activity reduces $\mathrm{BF}$ and $T_{\mathrm{m}}$ in the orofacial tissues. Furthermore, the simultaneous stimulation of the CST and LN increased $\mathrm{BF}$ and $\mathrm{VC}$; however, $T_{\mathrm{m}}$ was not increased in the lower lip (Fig. 6). These results suggest that the hemodynamics and $T_{\mathrm{m}}$ in the orofacial area could be susceptible to sympathetic activity. Modulation of the sympathetic nerve activity associated with stress and chronic pain, such as fibromyalgia, is known to induce changes in cardiovascular parameters, such as blood pressure and regional BF [27-29]. In addition, both vasoconstriction and decreased $T_{\mathrm{m}}$ during sympathoexcitation are thought to be associated with fibromyalgia in the tender points above the skin [30]. These observations suggest that disturbances in $\mathrm{BF}$ and $T_{\mathrm{m}}$ may play a role in the development of orofacial dysfunctions relevant to autonomic abnormalities.

Decreases in BF and $T_{\mathrm{m}}$ in the lower lip evoked by CST stimulation were inhibited significantly by LN stimulation (Fig. 6). This result indicates that the parasympathetic reflex vasodilation evoked by trigeminal afferent inputs compensates for the hypoperfusion of the $\mathrm{BF}$, which induces a decrease in $T_{\mathrm{m}}$ in the orofacial area. Elevated thermal conditions are thought to be involved in the functional properties of the orofacial tissues. Wound repair of the orofacial areas, such as the oral mucosa, has been reported to be faster than that in the dorsal skin [31, 32]; the thermosensitive transient receptor potential vanilloid 3 (TRPV3) is known to contribute to rapid wound healing in the oral epithelia [3]. Furthermore, the decreased sensitivity of the orofacial tissues (such as orofacial skin in lower lip and tongue tip) to hot and cold may be due to the high baseline $T_{\mathrm{m}}$ in the orofacial area $[1,33]$. These observations suggest that the trigeminal-parasympathetic reflex vasodilation for the maintenance of $T_{\mathrm{m}}$ may be involved in the functions of the oral epithelium and the sensory systems in the orofacial area under extensive stimuli during mastication, swallowing and speech.

\section{Conclusion}

In conclusion, our results suggest that parasympathetic vasodilation plays an important role in the maintaining the hemodynamics and $T_{\mathrm{m}}$ in the orofacial area, and that VIP may be involved in this response. The increase in BF evoked by parasympathetic reflex vasodilation, which induces an increase in the local $T_{\mathrm{m}}$, may be important for the compensation of hypoperfusion and for the decrease in $T_{\mathrm{m}}$, which are mediated by the CST. Further studies on the precise neural mechanisms, including molecular properties of the trigeminal-parasympathetic reflex vasodilation and the relationships between $T_{\mathrm{m}}$ and orofacial functions, will provide a better understanding of the functional properties with regard to the autonomic vasomotor responses in the orofacial area; in addition, the etiology of orofacial disorders, such as chronic pain and fibromyalgia related to the disturbances of the autonomic nervous system, may be further elucidated.

\section{Abbreviations}

ANOVA: Analysis of variance; a.u.: Arbitrary units; BF: Blood flow; CCD: Chargecoupled device; CST: Superior cervical sympathetic trunk; $\mathrm{C}_{6}$ : Hexamethonium; Fisher's PLSD: Fisher's protected least significant difference test; HR: Heart rate; iv: Intravenous; IX: Glossopharyngeal nerve root; LN: Lingual nerve; LSI: Laser speckle imaging; OG: Otic ganglion; ROI: Region of interest; SABP: Systemic arterial blood pressure; SCG: Superior cervical ganglion; SEM: Standard error of the mean; SN: Salivatory nuclei; $T_{m}$ : Local temperature; TG: Trigeminal ganglion; V: Trigeminal nerve root; VII: Facial nerve root; VIP: Vasoactive intestinal polypeptide; Vsp: Trigeminal spinal nucleus.

\section{Acknowledgements}

We appreciate the commitment of all participants of this study and their staff.

\section{Authors' contributions}

$\mathrm{HO}, \mathrm{MT}$, and $\mathrm{HI}$ make conception and design of research. $\mathrm{HO}, \mathrm{HI}, \mathrm{TS}$, and KM performed experiments. $\mathrm{HO}$ and $\mathrm{HI}$ analyzed data, interpreted results of experiments, prepared figures, and drafted manuscript. All authors edited and revised manuscript. All authors read and approved the final manuscript.

Funding

This study was supported, in part, by MEXT KAKENHI (No. 17K11650; to H. Ishii).

Availability of data and materials

All relevant data are within the paper.

Ethics approval and consent to participate

The experimental protocols were reviewed and approved by the Animal Ethics and Research Committee and conducted in accordance with the Regulations for the Care and Use of Laboratory Animals of the Health Sciences University of Hokkaido (No. 075). All the animals were cared for in accordance with the recommendations in the current National Research Council guide.

\section{Consent for publication}

Not applicable.

\section{Competing interests}

The authors declare that they have no competing interests, financial or otherwise, regarding this article.

\footnotetext{
Author details

${ }^{1}$ Division of Dental Anesthesiology, Department of Human Biology and Pathophysiology, School of Dentistry, Health Sciences University of Hokkaido, Ishikari-Tobetsu, Hokkaido, Japan. ${ }^{2}$ Division of Physiology, Department of Oral Biology, School of Dentistry, Health Sciences University of Hokkaido, 1757 Kanazawa, Ishikari-Tobetsu, Hokkaido 061-0293, Japan.
} 
Received: 2 February 2020 Accepted: 20 March 2020

Published online: 31 March 2020

\section{References}

1. Kim HK, Kim KS, Kim ME (2013) Influence of test site and baseline temperature on orofacial thermal thresholds. J Orofac Pain 27:263-270

2. Lemon CH (2017) Modulation of taste processing by temperature. Am J Physiol Regul Integr Comp Physiol 313:R305-R321

3. Aijima R, Wang B, Takao T, Mihara H, Kashio M, Ohsaki Y, Zhang JQ, Mizuno A, Suzuki M, Yamashita Y, Masuko S, Goto M, Tominaga M, Kido MA (2015) The thermosensitive TRPV3 channel contributes to rapid wound healing in oral epithelia. FASEB J 29:182-192

4. Gratt BM, Anbar M (1998) Thermology and facial telethermography: Part II. Current and future clinical applications in dentistry. Dentomaxillofac Radiol 27:68-74

5. Johnson JM, Minson CT, Kellogg DL Jr (2014) Cutaneous vasodilator and vasoconstrictor mechanisms in temperature regulation. Compr Physiol 4:33-89

6. Nemcovsky CE, Benvenisti A, Gazit E (1995) Variation of skin surface temperature over the masseter muscles in patients with myofascial pain following occlusal splint treatment. J Oral Rehabil 22:769-773

7. Ruocco I, Cuello AC, Parent A, Ribeiro-da-Silva A (2002) Skin blood vessels are simultaneously innervated by sensory, sympathetic, and parasympathetic fibers. J Comp Neurol 448:323-336

8. Uchida Y, Atsumi K, Takamata A, Morimoto K (2019) The effect of menstrual cycle phase on foot skin temperature during mild local cooling in young women. J Physiol Sci 69:151-157

9. Kaji A, Maeda T, Watanabe S (1991) Parasympathetic innervation of cutaneous blood vessels examined by retrograde tracing in the rat lower lip. J Auton Nerv Syst 32:153-158

10. Izumi H (1995) Reflex parasympathetic vasodilatation in facial skin. Gen Pharmacol 26:237-244

11. Ishii H, Niioka T, Izumi H (2007) Parasympathetic vasodilator fibers in masseter muscle. J Oral Biosci 49:163-172

12. Kaji A, Shigematsu H, Fujita K, Maeda T, Watanabe S (1988) Parasympathetic innervation of cutaneous blood vessels by vasoactive intestinal polypeptide-immunoreactive and acetylcholinesterase-positive nerves: histochemical and experimental study on rat lower lip. Neuroscience 25:353-362

13. Hardebo JE, Suzuki N, Ekblad E, Owman C (1992) Vasoactive intestinal polypeptide and acetylcholine coexist with neuropeptide $Y$, dopamine- $\beta$ hydroxylase, tyrosine hydroxylase, substance $P$ or calcitonin gene-related peptide in neuronal subpopulations in cranial parasympathetic ganglia of rat. Cell Tissue Res 267:291-300

14. Niioka T, Ishii H, Izumi H (2009) Involvement of vasoactive intestinal polypeptide in the parasympathetic vasodilatation of the rat masseter muscle. Arch Oral Biol 54:909-916

15. Sato T, Ishii H (2015) Differences in control of parasympathetic vasodilation between submandibular and sublingual glands in the rat. Am J Physiol Regul Integr Comp Physiol 309:R1432-R1438
16. Izumi H, Karita K (1992) Somatosensory stimulation causes autonomic vasodilatation in cat lip. J Physiol 450:191-202

17. Ishii H, Niioka T, Sudo E, Izumi H (2005) Evidence for parasympathetic vasodilator fibres in the rat masseter muscle. J Physiol 569:617-629

18. Sudo E, Ishii H, Niioka T, Hirai T, Izumi H (2009) Parasympathetic vasodilator fibers in rat digastric muscle. Brain Res 1302:125-131

19. Mizuta K, Karita K, Izumi H (2000) Parasympathetic reflex vasodilatation in rat submandibular gland. Am J Physiol Regul Integr Comp Physiol 279:R677-R683

20. Izumi H, Ito Y (1998) Sympathetic attenuation of parasympathetic vasodilatation in oro-facial areas in the cat. J Physiol 510:915-921

21. Ishii H, Niioka T, Watanabe H, Izumi H (2007) Inhibitory effects of excess sympathetic activity on parasympathetic vasodilation in the rat masseter muscle. Am J Physiol Regul Integr Comp Physiol 293:R729-R736

22. Ishii $H$, Sato $T$ (2017) Interactions between $\beta$-adrenergic vasodilation and cervical sympathetic nerves are mediated by $a_{2}$-adrenoceptors in the rat masseter muscle. J Physiol Sci 67:699-709

23. Niioka T, Ishii H, Izumi H (2009) Regional differences in blood flow variation in rat masseter muscle. Arch Oral Biol 54:1022-1028

24. Ishii H, Sato T, Izumi H (2014) Parasympathetic reflex vasodilation in the cerebral hemodynamics of rats. J Comp Physiol B 184:385-399

25. Stern MD, Lappe DL, Bowen PD, Chimosky JE, Holloway GA Jr, Keiser HR, Bowman RL (1977) Continuous measurement of tissue blood flow by laser-Doppler spectroscopy. Am J Physiol 232:H441-H448

26. Szabo E, Csaki A, Boldogkoi Z, Toth Z, Koves K (2015) Identification of autonomic neuronal chains innervating gingiva and lip. Auton Neurosci 190:10-19

27. Delcanho RE, Kim YJ, Clark GT (1996) Haemodynamic changes induced by submaximal isometric contraction in painful and non-painful human masseter using near-infra-red spectroscopy. Arch Oral Biol 41:585-596

28. Kulshreshtha P, Deepak KK (2013) Autonomic nervous system profile in fibromyalgia patients and its modulation by exercise: a mini review. Clin Physiol Funct Imaging 33:83-91

29. Maekawa K, Clark GT, Kuboki T (2002) Intramuscular hypoperfusion, adrenergic receptors, and chronic muscle pain. J Pain 3:251-260

30. Jeschonneck M, Grohmann G, Hein G, Sprott H (2000) Abnormal microcirculation and temperature in skin above tender points in patients with fibromyalgia. Rheumatology 39:917-921

31. Sciubba JJ, Waterhouse JP, Meyer J (1978) A fine structural comparison of the healing of incisional wounds of mucosa and skin. J Oral Pathol 7:214-227

32. Szpaderska AM, Zuckerman JD, DiPietro LA (2003) Differential injury responses in oral mucosal and cutaneous wounds. J Dent Res 82:621-626

33. Oono Y, Wang K, Atis ES, Arendt-Nielsen L (2013) Thermal application modulates orofacial somatosensory perception in healthy men and women. Clin Neurophysiol 124:581-588

\section{Publisher's Note}

Springer Nature remains neutral with regard to jurisdictional claims in published maps and institutional affiliations.
Ready to submit your research? Choose BMC and benefit from:

- fast, convenient online submission

- thorough peer review by experienced researchers in your field

- rapid publication on acceptance

- support for research data, including large and complex data types

- gold Open Access which fosters wider collaboration and increased citations

- maximum visibility for your research: over 100M website views per year

At BMC, research is always in progress.

Learn more biomedcentral.com/submissions 